EPJ Web of Conferences 41, 04004 (2013)

DOI: $10.1051 /$ epjconf/20134104004

(C) Owned by the authors, published by EDP Sciences, 2013

\title{
Non-perturbative four-wave mixing in InSb with intense off- resonant multi-THz pulses
}

\author{
B. Mayer ${ }^{1}$, F. Junginger ${ }^{1}$, C. Schmidt ${ }^{1}$, S. Mährlein ${ }^{1}$, O. Schubert ${ }^{1,2}$, A. Pashkin ${ }^{1}$, R. Huber ${ }^{1,2}$, \\ and A. Leitenstorfer ${ }^{1}$ \\ ${ }^{1}$ Department of Physics and Center for Applied Photonics, University of Konstanz, \\ Universitätsstr. 10, 78464 Konstanz, Germany \\ ${ }^{2}$ Department of Physics, University of Regensburg, Universitätsstr. 31, 93053 Regensburg, Germany
}

\begin{abstract}
High-field multi-THz pulses are employed to analyze the coherent nonlinear response of the narrow-gap semiconductor InSb which is driven off-resonantly. Fieldresolved four-wave mixing signals manifest the onset of a non-perturbative regime of Rabi flopping at external amplitudes above $5 \mathrm{MV} / \mathrm{cm}$ per pulse. Simulations based on a two-level quantum system confirm these experimental results.
\end{abstract}

Two-dimensional spectroscopy has been a powerful tool to examine spectral correlations and ultrafast dynamics in condensed-matter systems. For example, studies in the near- (NIR) and midinfrared (MIR) spectral region [1] have provided insight into molecular structures of proteins [2]. At yet lower $\mathrm{THz}$ frequencies two-dimensional spectroscopy has been anticipated to provide access to fundamental low-energy excitations such as inter-molecular motion in water [3] or gap dynamics in superconductors [4].

Recently, a promising $\mathrm{THz}$ multi-wave mixing has been suggested [5]. These lead-off experiments exploiting field amplitudes of the order of $1 \mathrm{MV} / \mathrm{cm}$ have been limited to model systems with especially large dipole moments. It has been shown that Rabi oscillations can be driven resonantly in semiconductors with intense optical pulses [6,7]. The latest development of an ultraintense laser source for MIR pulses with peak fields exceeding $100 \mathrm{MV} / \mathrm{cm}$ paves the way towards a systematic study of extreme $\mathrm{THz}$ nonlinearities $[8,9]$. Based on the electro-optic detection scheme, the time trace of the carrier field can be retrieved with absolute amplitude and phase.

Here, a high-field $\mathrm{THz}$ laser system is employed to study the nonlinear response of bulk semiconductors using a two-dimensional spectroscopy scheme [5]. We show that in spite of the excitation far below the interband resonance extreme $\mathrm{THz}$ electric fields are able to coherently drive a system close to population inversion.

Our THz multi-wave mixing experiment is performed in the narrow-gap semiconductor indium antimonide $\mathrm{InSb}\left(E_{\mathrm{g}} / \mathrm{h}=41.1 \mathrm{THz}\right)$ at room temperature. An undoped (100)-oriented single crystal has been mechanically polished to a thickness of $30 \mu \mathrm{m}$ facilitating $\mathrm{THz}$ transmission. Intense $\mathrm{THz}$ transients centered at $25 \mathrm{THz}$ are obtained by difference frequency mixing of near-infrared pulse trains in a GaSe emitter. All relevant frequency components are located well below the fundamental band gap of InSb. In contrast to previous studies [5], the generation of double pulses is implemented in our setup by splitting the $\mathrm{THz}$ beam after the emitter crystal to preclude nonlinear mixing effects of two beams within the emitter itself. Two equally intense transients are obtained via a Au-coated

This is an Open Access article distributed under the terms of the Creative Commons Attribution License 2.0, which permits unrestricted use, distribution, and reproduction in any medium, provided the original work is properly cited. 
Ge substrate. The transmitted pulse is delayed by a linear stage. Both beams are tightly focused onto the sample. Emerging linear and nonlinear fields are collected with large numerical aperture, directed onto a $140-\mu \mathrm{m}$-thick GaSe sensor, and electro-optically sampled by 8 fs-short near-infrared gating pulses.

The total transmitted field $E_{12}$ is detected as a function of the electro-optic sampling delay time $t$ and the relative temporal offset $\tau$ between the THz pulses. We retrieve the nonlinear signal $E_{\mathrm{NL}}$ by subtracting the linear contributions $\left(E_{1}\right.$ and $\left.E_{2}\right)$ from the total response: $E_{\mathrm{NL}}=E_{12}-E_{1}-E_{2}$. A twodimensional Fourier transform (FT) of $E_{\mathrm{NL}}$ enables the separation of various nonlinear contributions, such as pump-probe signals and four-wave mixing (FWM) processes [5]. The inverse FTs of selected regions in frequency space then individually display the temporal hallmark of the nonlinearity. In particular the FWM response is sensitive to the respective phases of both $\mathrm{THz}$ pulses and carries information about the coherence induced in the system. Therefore, it is free of influence of incoherent processes related e.g. to carrier multiplication.

For an experimental study of the field dependence of the FWM signal external peak fields of $2 \mathrm{MV} / \mathrm{cm}, 3.5 \mathrm{MV} / \mathrm{cm}$ and $5.3 \mathrm{MV} / \mathrm{cm}$ per few-cycle pulse are applied. An oval-shaped envelope of the time domain FWM signal is detected for the lower electric field regime. This signal is identical to the cross-correlation function of both pulses (Figure 1(a)) as expected in perturbative nonlinear optics far from resonance. For higher peak electric fields a deviation from the symmetric profile is observed, leading towards an asymmetric S-shaped signal for external peak fields of $3.5 \mathrm{MV} / \mathrm{cm}$ (Figure 1(b)). At the maximum field of $5.3 \mathrm{MV} / \mathrm{cm}$ even a splitting of the FWM signal is induced, indicating an extremely nonlinear regime (Figure 1(c)).
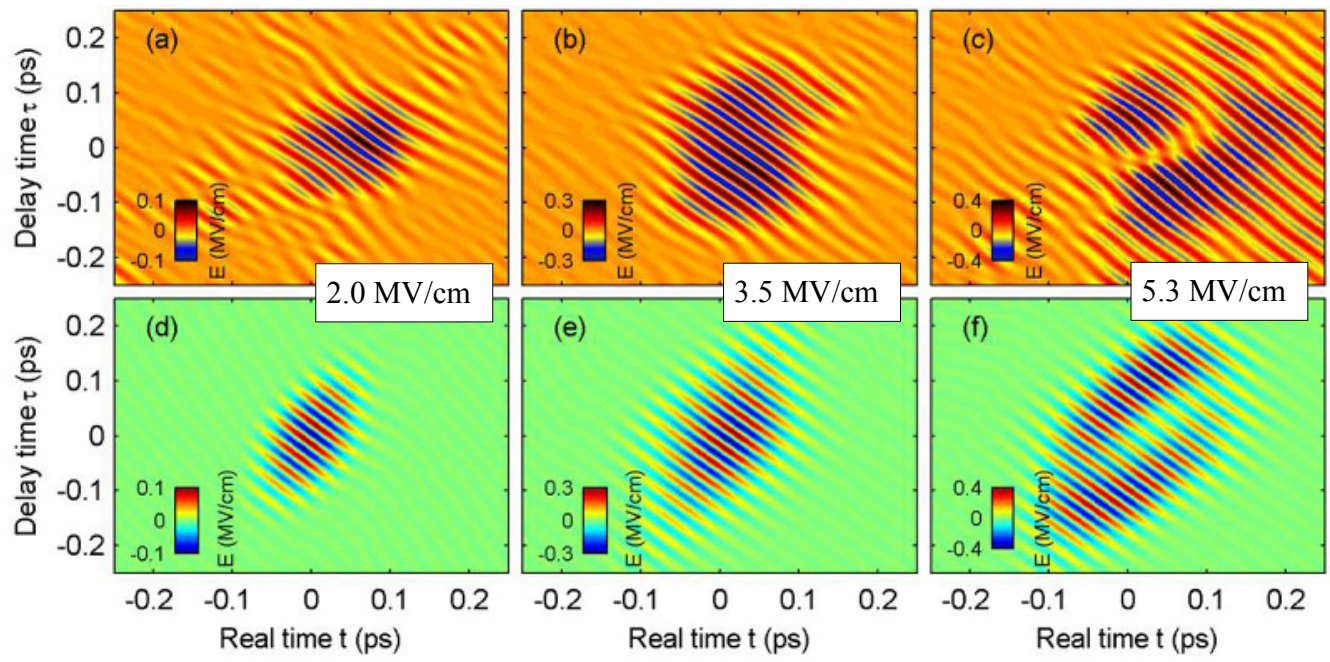

Fig. 1. (a-c) Two-dimensional FWM signatures with external peak fields of the driving transient of $2 \mathrm{MV} / \mathrm{cm}, 3.5 \mathrm{MV} / \mathrm{cm}$ and 5.3 MV/cm, respectively. (d-f) Simulated FWM data reproducing the essential features of the corresponding measured signals above.

For a better understanding of these results we analyze a two-level system as a simplified model, describing the interband resonance in InSb which is driven by an off-resonant $\mathrm{THz}$ transient with few optical cycles. This simulation is based on a system of Maxwell-Bloch equations [10], which are solved numerically without applying the rotating-wave and slowly-varying-envelope approximations. A set of time domain FWM signals for different field strengths is calculated and shown in Figures 1(d)-(f). The transition dipole moment $\mu_{12}=2.4$ e $\AA$ and the density of the two-level systems $\mathrm{N}=2.9 \cdot 10^{20} \mathrm{~cm}^{-3}$ were selected to provide the best agreement concerning the shape and intensity of the experimentally obtained FWM signatures. The essential features observed in the experiment are well reproduced in the simulation. 
For a qualitative physical understanding of the splitting observed in the FWM signal for high peak electric fields, one can study the polarization response of a two-level system driven by two temporally overlapping $\mathrm{THz}$ transients $(\tau=0 \mathrm{ps})$. Figure 2(a) shows a simulation of the timeresolved dielectric polarization for different peak electric fields $E_{\mathrm{m}}$ of the driving pulses. The plot on the right hand side indicates field-dependent maximal population inversion $w_{\max }$. For moderate fields $w_{\max }$ remains negative indicating a perturbative response of the two-level system. The shape of the polarization follows the field profile of the driving field (Figure 2(a) bottom) and the deviation of the Bloch vector from the ground state is minor (Figure 2(b)). For a high peak electric field in excess of $5 \mathrm{MV} / \mathrm{cm}$, the system is promoted almost to the limit of a complete population inversion (Figures 2(a),(c)). In this regime, the corresponding polarization response is minimal leading to the observed splitting in the center of the FWM signal where the driving field reaches its maximum. Here, our high-field $\mathrm{THz}$ transients induce a Rabi splitting comparable to the large detuning of 18 $\mathrm{THz}$ between driving field and the fundamental interband transition of InSb.
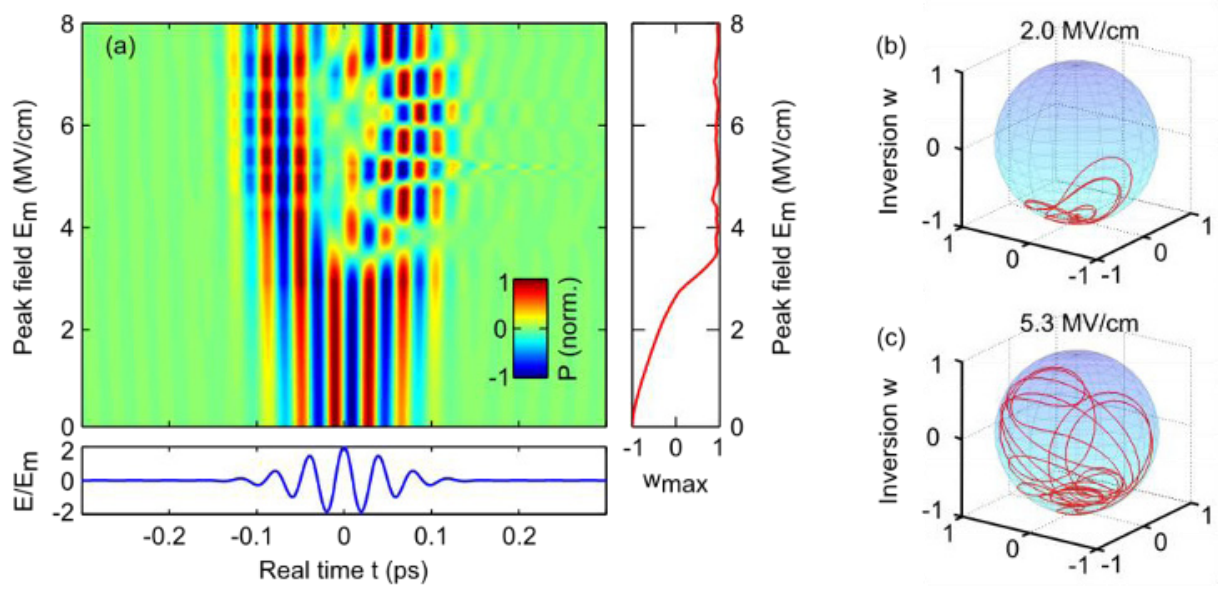

Fig. 2. (a) Normalized polarization response of the two-level system as a function of the real time $t$ and the peak electric field $E_{\mathrm{m}}$ for $\tau=0$ ps. Right hand side: Maximum inversion $w_{\max }$ as a function of $E_{\mathrm{m}}$. Bottom: Driving field of the THz transient. $(\mathrm{b}, \mathrm{c})$ Corresponding pathways of the Bloch vector for a moderate $\left(E_{\mathrm{m}}=2 \mathrm{MV} / \mathrm{cm}\right)$ and a high $\left(E_{\mathrm{m}}=5.3 \mathrm{MV} / \mathrm{cm}\right)$ peak electric field.

In conclusion, ultraintense few-cycle $\mathrm{THz}$ pulses are generated to drive bulk InSb far off the band gap providing a direct access to the coherent dynamics of the interband polarization. A new regime with a non-perturbative response is observed proving the high potential of high-field multi- $\mathrm{THz}$ technology.

1. M. Cho, Chem. Rev. 108, 1331 (2008)

2. R. M. Hochstrasser, Proc. Natl. Acad. Sci USA 104, 14190 (2007)

3. T. Yagasaki, J. Ono, S. Saito, J. Chem. Phys. 131, 164511 (2009)

4. T. Papenkort, T. Kuhn, V. M. Axt, Phys. Rev. B 78, 132505 (2008)

5. W. Kuehn, K. Reimann, M. Woerner, T. Elsaesser, J. Chem. Phys. 130, 164503 (2009)

6. S. T. Cundiff, A. Knorr, J. Feldmann, S. W. Koch, E. O. Göbel, H. Nickel, Phys. Rev. Lett. 87,057401 (2001)

7. S. Leinß, T. Kampfrath, K. v. Volkmann, M. Wolf, J. T. Steiner, M. Kira, S. W. Koch, A. Leitenstorfer, R. Huber, Phys. Rev. Lett. 101, 246401 (2008)

8. A. Sell, A. Leitenstorfer, R. Huber, Opt. Lett. 33, 2767 (2008)

9. F. Junginger, A. Sell, O. Schubert, B. Mayer, D. Brida, M. Marangoni, G. Cerullo, A. Leitenstorfer, R. Huber, Opt. Lett. 35, 2645 (2010)

10. R. W. Ziolkowski, J. M. Arnold, and D. M. Gogny, Phys. Rev. A 52, 3082 (1995) 